\title{
Application of on-line quality control for salvianolic acid $B$ by near infrared spectroscopy
}

\author{
Jin-Wei Zhang*, Yan Liu, Wei-Wei Liu, Yan-Ying Zhang
}

Tasly Modern TCM Resources Co., LTD., Tianjin, China; tjvv@163.com

Received 11 August 2009; revised 23 September 2009; accepted 22 October 2009

\begin{abstract}
OBJECTIVE: To study and establish quality control model of the Salvianolic Acid B by Near Infrared Spectroscopy (NIRS), and to realize on-line quality control of extracting and purifying processes of industrial scale herbal product manufacturing. METHOD: NIR chromatography was obtained from on-line NIR detection of extracting process and purifying process. HPLC analysis was carried out to determine the contents of salvianolic acid B. Partial Least Squares Regression (PLS) was used to establish the model between the information between NIRS and HPLC. RESULTS: For extracting model: the optimum Near Infrared (NIR) wavelength range was 9815- $5430 \mathrm{~cm}^{-1}, \mathrm{R}=0.9784$, RMSEC $=0.258$; for purifying model: the optimum NIR wavelength range was $9815-5430 \mathrm{~cm}^{-1}, \mathrm{R}=0.9776$, RMSEC $=4.02$. The average relative error was $<5 \%$. CONCLUSION: NIR technique is applicable for on-line quality control in production of salvianolic acid $B$.
\end{abstract}

Keywords: NIR; Salvianolic Acid B; On-Line Quality Control; Industrial Scale

\section{INTRODUCTION}

Real-time control of contents has ground to gain in controlling mode of Chinese medicine manufacturing in China, as the very traditional techniques remain the mainstream. Off-line tests do not meet the on-line control demands for production process. On-line test is the solution to quality consistency, which is still the bottle-neck in manufacturing of Chinese medicine.

Near infrared (NIR) Spectroscopy technology develops rapidly in recent years as a fast analytical technology. It shows the substances and structures of the tested samples indirectly. The procedure of acquiring information by NIR chromatography is easy and low-cost. It does not require complicated pre-treatment of the samples but still meets the requirement of on-line testing.
As the chemistry metrology and computer technology develop, NIR has already been widely adopted in agriculture and petroleum industries [1-2]. In 2002, USFDA approved NIR to be one of the standard measurement methods. In 2005, "China Pharmacopoeia" listed "NIR Spectroscopy instruction" in [3]. NIR Spectroscopy technology turns out to be a research hotspot in on-line testing in Chinese medicine industry, especially in manufacturing process recently. Some of the experts use NIR to measure the content of Tanshinone and Salvianolic acid B in Danshen liquid distilling process [4] while some others use NIR in quantitative analysis of Gardenia herbs distilling and Notoginsenosides [5-6]. NIR is also reported to be used in real-time analysis of CoptisRoot and BittetOrange extract purification processes [7-8]. But these are limited in laboratory scale simulated manufacture research, but not yet in manufacture scale distilling or purification of TCM.

This research covers the key working procedures in manufacturing of Salvianolic acid B, including distilling and purification, and focuses on concentration of Salvianolic acid B, which is the main effective ingredient in aqueous extract and elute. On-line NIRS chromatography charts are collected and chemometrical calculation is done based upon data from HPLC testing. Models for content analysis of Salvianolic acid B in distilling and purification processes are established in order to facilitate real-time quality monitoring in manufacturing of Salvianolic acid B.

\section{MATERIALS AND METHODS}

\subsection{Instruments and Materials}

\subsubsection{Instruments}

ANTARIS FT-NIR(Thermo), optical fiber and TQ Analyst software; Agilent 1100 HPLC; 500L Multifunctional distill tank (Tianjin Tasly Modern TCM Resources co., LTD); 150L Stainless steel chromalography columns (Tianjin Tasly Modern TCM Resources co., LTD).

\subsubsection{Materials}

Danshen (From Shangluo, Shanxi, China; identified as by 
Quality Control Department of Tianjin Tasly Modern TCM Resources co., LTD);

Salvianolic acid B reference standard (from National Institute For The Control of Pharmaceutical and Biological);

Methanol, acetonitrile (chromatographical grade) (Merk);

Methanoic acid is (Analytical grade; Tianjin Chemical Reagent Co., LTD).

\subsection{Rationale and Method}

\subsubsection{Rationale (Moved To the Introduction Section)}

The NIR is electromagnetic wave between VIS and MIR. ASTM (American Society of Testing Materials) defined The area of NIR chromatography as $780 \sim 2526 \mathrm{~nm}$ $(12820 \sim 3959 \mathrm{~cm}-1)$ [9]. The wavelength of NIR covers frequencies of organic molecule groups with hydrogen $(\mathrm{OH}, \mathrm{NH}$ and $\mathrm{CH})$. Scanning organic samples with NIR produces information about the organic molecules with hydrogen. Absorption and maximum absorption wavelength differ from each other with different groups or same group in different chemical constitutions, and therefore, the chromatography bears information of chemical structures, which ensures its application in analysis of bioactive ingredients [10]. Because of the width and overlapping of NIR absorption ranges, application of chemometrics is a necessity in extraction of chromatographic information. In this research, HPLC test is applied for content analysis of entities, while PLS regression applied for establishment of model between NIR information and content analysis information for on-line testing and quality monitoring of Salvianolic acid B.

\subsubsection{Methods}

\section{1) Research system}

Weigh 50kg Danshen, put into the extracting tank-500L, add 5.5 times of water and start decoction; after 1 hour, export extract solution then add triple water to extracting waste; after $0.5 \mathrm{~h}$, export extract solution. Mix and prepare the extract solutions for separation with polyamide resins. In the process of extracting and refine, we collect chromatography data at regular intervals; test samples by HPLC. The extract and refine devices were shown in the following illustration:

2) On-line collection of NIR chromatography data

2 parallel chromatographic charts are collected at $2-5 \mathrm{~min}$ intervals in the process of extracting and refining. Samples for HPLC tests are collected at the same time of collecting NIR chromatography. Samples and NIR chromatography charts are coded correspondingly.

NIR chromatography is collected at scanning scope of $10000-4000 \mathrm{~cm}^{-1}$, scanning number of 32 , and resolution of $8 \mathrm{~cm}^{-1}$. The NIR chromatography of Danshen extract and elutriant is shown in the following illustration 3-4.

In the process of collecting NIR chromatography, air

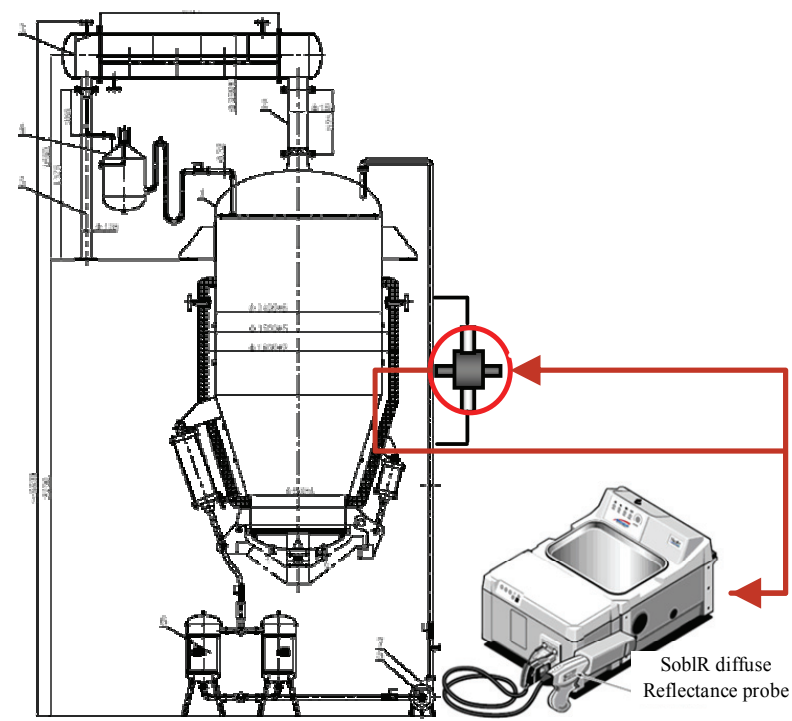

Figure 1. Extracting equipment of Salvianolic acid B.

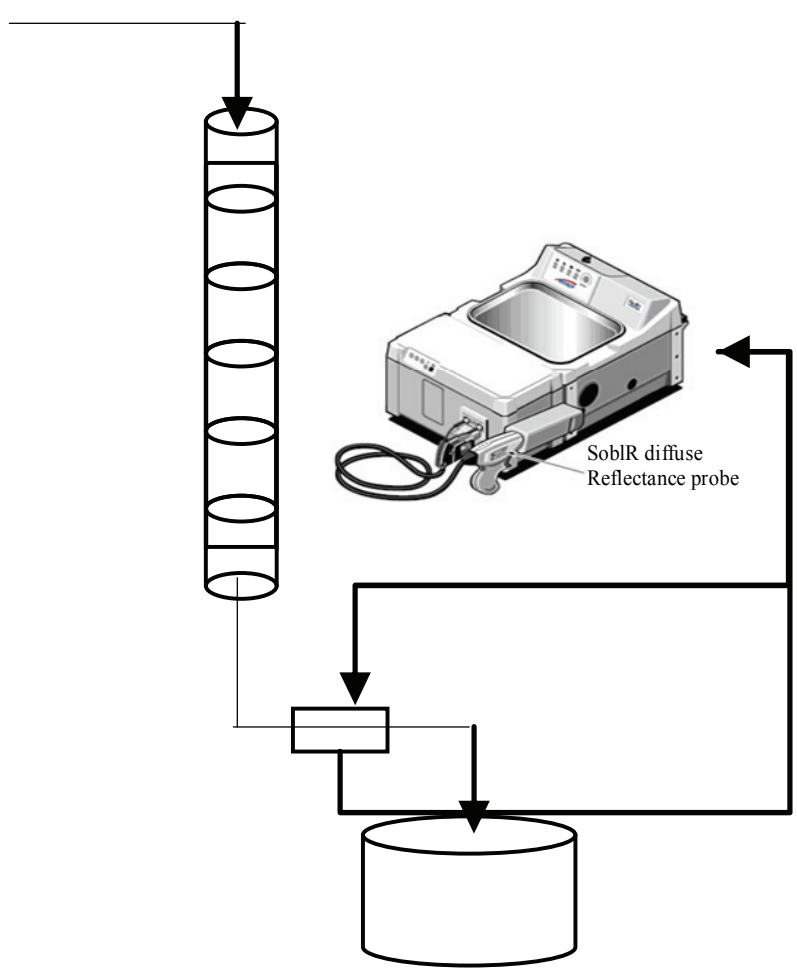

Figure 2. Separation equipment of Salvianolic acid B.

bubble in the flow cell is a problem in the process of experiment, as it is directly influence the absorption and emission of signals, resulting in irregular peaks in chromatography and serious interference of chromatography information. Measures are taken with equipment and process till the solution of the issue.

3) Testing method of Salvianolic acid B

Chromatographic column: Agilent ZORBAX SB-C18, 5 um, $4.6 * 250 \mathrm{~mm}$; mobile phase: methanol-acetonitrile- 


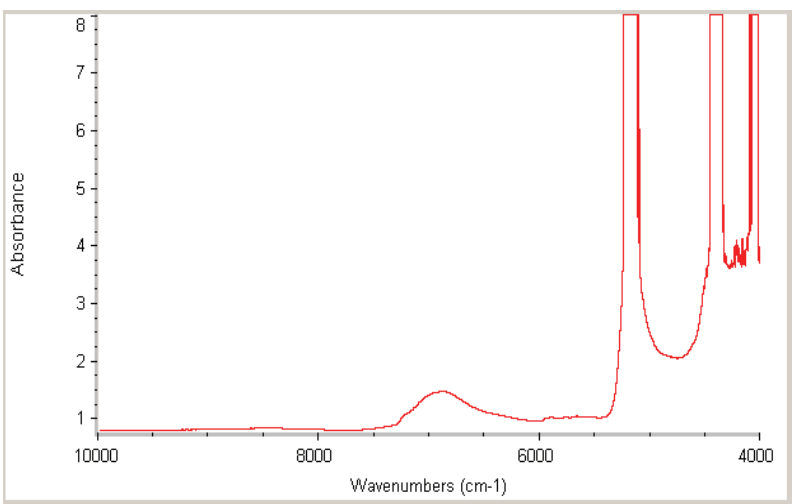

Figure 3. NIR chromatography of salvianolic acid B extract.

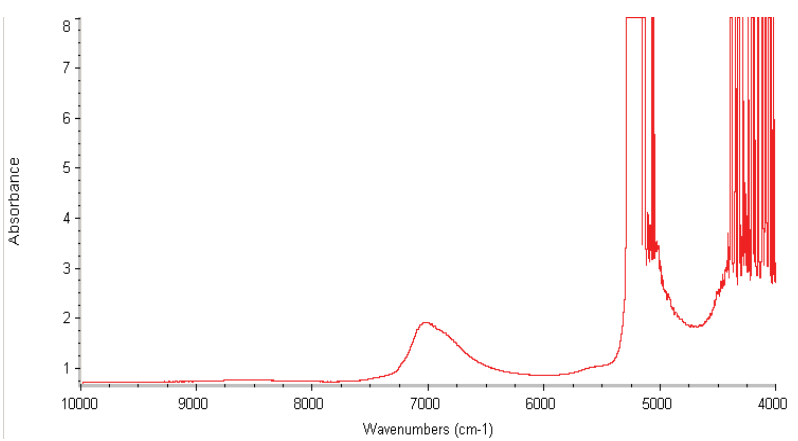

Figure 4. NIR chromatography of salvianolic acid elutriant.

methanoic acid-water $=30: 10: 1: 59$; detection wavelength: $286 \mathrm{~nm}$; flow rate: $1 \mathrm{ml} / \mathrm{min}$; column temp: $30^{\circ} \mathrm{C}$; injection: $5 \mathrm{ul}$; preparation of reference substance solution: weigh accurately salvianolic acid B reference, and add it to the $75 \%$ methanol to make methanol solution of $0.14 \mathrm{mg} / \mathrm{ml}$; preparation of test solution: weigh accurately extract solution and elutriant, add it to the $75 \%$ methanol and dilute to $10 \mathrm{ml}$, shake till well mixed, and then filtrate to the $0.45 \mathrm{um}$. Filtrate is the test solution.

\section{RESULTS AND DISCUSSIONS}

\subsection{Pretreatment of Chromatography Data}

In the process of collecting chromatography, environmental changes produce baseline excursion, while random noise of sample background produce influences on calibration results. Pretreatment of the chromatography data is conducted for the issue. Factorial analysis of various pretreatment methods and statistical factors in extracting and refining models are listed in Table $\mathbf{1}$ and Table 2 .

The methods of pretreatment are MSC, SNV, firstorder differential equation, second-order differential equation, S-G (Savitzky-Golay) smooth and Norris Derivative Filter smooth etc. 1st derivative +Norris is selected as the pretreatment for both extract and refine model accordingly.
Table 1. Effect of various pretreatment methods on extracting model.

\begin{tabular}{lccc}
\hline $\begin{array}{l}\text { Method of the chromatography } \\
\text { pretreatment }\end{array}$ & $\mathrm{R}$ & RMSEC & RMSECV \\
\hline Original chromatography & 0.9581 & 0.358 & 0.389 \\
MSC & 0.8094 & 0.733 & 0.807 \\
MSC+ 1st derivative & 0.9169 & 0.498 & 0.566 \\
MSC+ 2nd derivative & 0.9055 & 0.530 & 0.911 \\
MSC+1st derivative +Norris & 0.8978 & 0.550 & 0.612 \\
MSC+2nd derivative +Norris & 0.8907 & 0.568 & 0.666 \\
SNV & 0.8131 & 0.727 & 0.800 \\
SNV+1st derivative & 0.9179 & 0.496 & 0.743 \\
SNV+2nd derivative & 0.9064 & 0.528 & 0.906 \\
SNV+1st derivative +Norris & 0.8988 & 0.547 & 0.610 \\
SNV+2nd derivative +Norris & 0.8906 & 0.568 & 0.666 \\
1st derivative +Norris & 0.9784 & 0.258 & 0.286 \\
2nd derivative +Norris & 0.9574 & 0.361 & 0.430 \\
1st derivative +S-G & 0.9746 & 0.279 & 0.378 \\
2nd derivative +S-G & 0.9110 & 0.515 & 0.761 \\
\hline
\end{tabular}

Table 2. Effect of various pretreatment methods on purifying model.

\begin{tabular}{lccc}
\hline $\begin{array}{l}\text { Method of the chromatography } \\
\text { pretreatment }\end{array}$ & R & RMSEC & RMSECV \\
\hline Original chromatography & 0.6681 & 14.9 & 15.8 \\
MSC & 0.7782 & 12.6 & 13.4 \\
MSC+1st derivative & 0.9657 & 5.27 & 7.09 \\
MSC+2nd derivative & 0.9761 & 4.38 & 11.3 \\
MSC+1st derivative +Norris & 0.9635 & 5.39 & 6.95 \\
MSC+2nd derivative +Norris & 0.9524 & 6.15 & 7.05 \\
SNV & 0.9313 & 7.34 & 8.18 \\
SNV+1st derivative & 0.9624 & 5.49 & 6.99 \\
SNV+2nd derivative & 0.9730 & 4.65 & 11.5 \\
SNV+1st derivative +Norris & 0.9551 & 5.97 & 6.80 \\
SNV+2nd derivative +Norris & 0.9500 & 6.28 & 7.06 \\
1st derivative +Norris & 0.9776 & 4.02 & 4.42 \\
2nd derivative +Norris & 0.9668 & 5.26 & 6.09 \\
1st derivative +S-G & 0.9733 & 4.56 & 5.53 \\
2nd derivative +S-G & 0.9760 & 4.46 & 9.59 \\
\hline
\end{tabular}

\subsection{Selection for the Best Principal Factors}

In regression for the models with PLS, rational selection of principal factors is critical to avoid "over fitting". Through LOOCV (leave-one-out cross-validation), we inspect the influence principal factors to RMSECV (Root-Mean-Square Error of Cross-Validation). The results for extracting and refining models are in Figure 5 
and Figure 6.

When the principal factor number is 5 , the RMSECV is 0.286 and reaches pleateau phase. It is decided the principal factor number is 5. From Figure 6, RMSECV reaches peak at 4.42 when principal factor number of refining model is 6 .

\subsection{Selection of Chromatography Scope}

The decision of chromatography scope is the most difficult step in the quantitative analysis model of NIR, as the calculation is not well established in chemometrics. The wave band of $9815-5430 \mathrm{~cm}^{-1}$ is validated as the optimal chromatography scope with optimized correlation coefficient and forecast effects.

\subsection{Establishment of Models}

Salvianolic acid B contents in 6 batches are analyzed, while 473 experiment samples (222 from extracting process and 251 from refining process) are collected. First-order differential equation is the statistical method in establishment of Salvianolic acid B models through PLS. According to the result of cross-validation: extracting model: the best principal factor number is 5, correlation coefficient $\mathrm{R}=0.784$, $\mathrm{RMSEC}=0.258$; refining model: the best principal factor number is 6, correlation coefficient $\mathrm{R}=0.9776, \mathrm{RMSEC}=4.02$. The correlation coefficients of predicted results and test results are shown as following table:

\subsection{Evaluation of Prediction}

In order to validate the predict effect of the model or REF, a batch with the same manufacturing conditions, is produced, and 19 extracting samples and 33 refining samples are collected respectively for the validation of the models. The trend in predicted values and test values of Salvianolic acid B is shown as following:

Obvious from Figures 9 and 10, predicted values match test values in a constant and steady style with exceptions in few outliers. The relative error is less than 5 between predictive values and true values $(3.2 \%$ in extracting model and $4.8 \%$ in refining model). Abnormal data points may be result of several facts. First of all, NIR test requires tested content to be at least $0.1 \%$. Very low target concentration leads to more errors in measurement. The on-line nature of the measurement also contributes in the errors, as test liquid is in constant movement in pipeline, and instability, as well as formation of bubbles in circulation pool is inevitable.

\section{CONCLUSIONS}

1) NIR (near infrared) Spectroscopy technology have many strong points including convenience, time effectiveness, cost effectiveness, and environment friendliness. On the basis of the research and model, it is feasible to

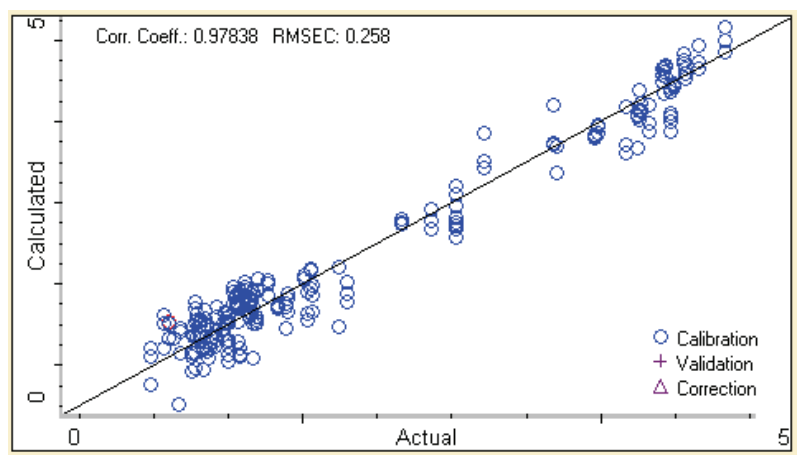

Figure 7. Correlation of salvianolic acid B's content between actual and calculated.

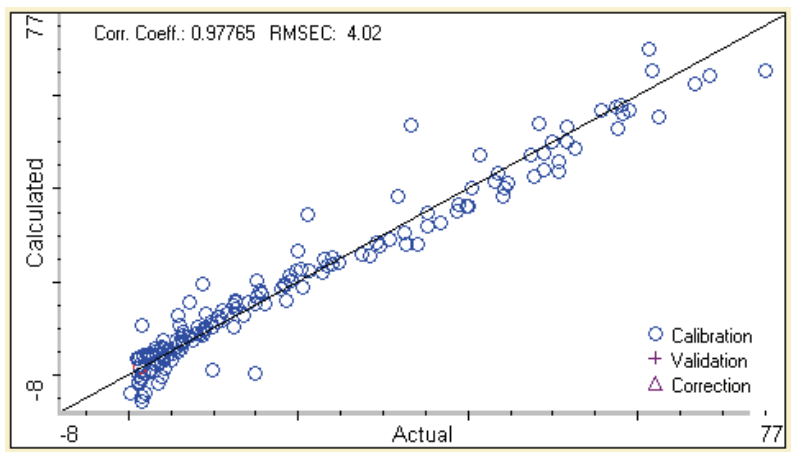

Figure 8. Correlation of salvianolic acid B's content between actual and calculated.

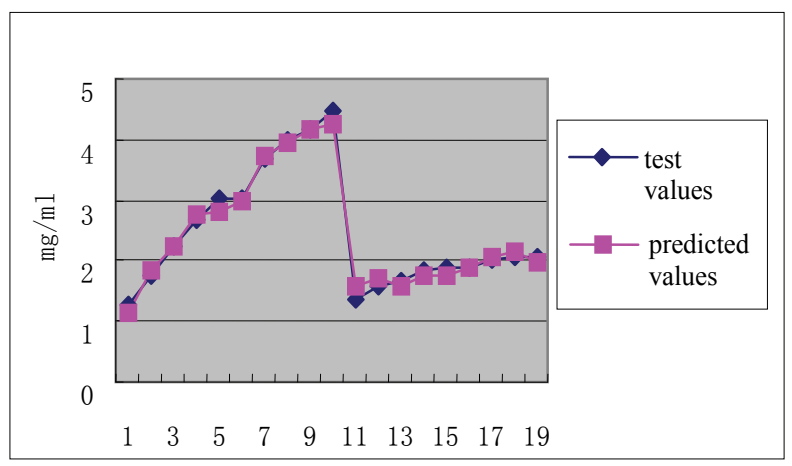

Figure 9. Predicted and test values of Salvianolic acid B content.

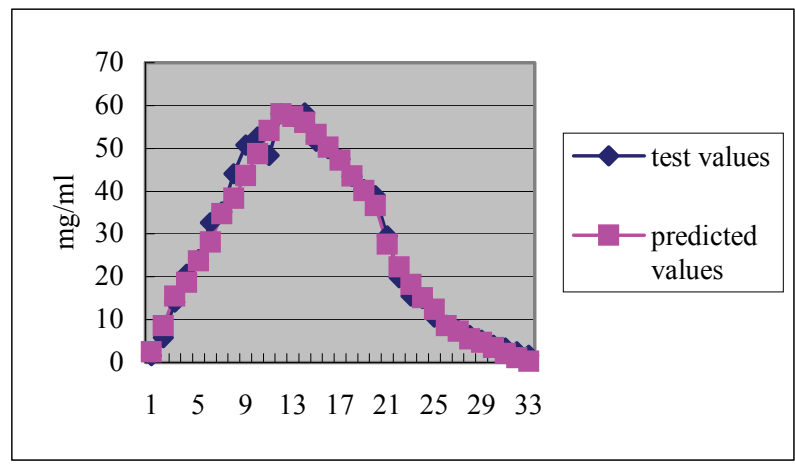

Figure 10. Predicted and test values of Salvianolic acid B content. 
implement on-line testing and quality monitoring as the prediction effects of the extraction and refinement models are acceptable in manufacturing of Salvianolic acid B.

2) Pretreatment of the chromatography data and principle factor number has influence on the accuracy of the model. Analysis and comparison of various conditions and is necessary in selection of the principal factors.

3) Extremely low concentration of target entity, instability and bubble formation in the current have influence over collection of chromatography data and over accuracy of the model in prediction. Necessary measurement should be taken to reduce the influences.

\section{REFERENCES}

[1] L. G. Weyer, (1985) Applied spectroscopy. [J]. Reviews, 21 (1-2), 1-8.

[2] G. A. Lang, (1994) NIRs monitor critical gasoline parameters. [J]. Hydrocarbon Process, 72(2), 69-72.

[3] N. Wang and H. Wei, (2007) Application potentials of on-line near infrared spectroscopy technology in manufacturing of traditional Chinese medicine. [J]. Journal of Shandong University of Traditional Chinese Medicine, 31(4), 350-353.

[4] L. J. Ni, X. H. Shi, X. J. Gao, etc. (2004) Research on the application of on-line detection and analytical technique by NIR in quality control of water extracting process of Salvia miltiorrhiza. [J]. Chinese Pharmaceutical Journal, 39(8), 628-630.

[5] S. K. Yan, L. A. Luo, Y. M. Wang, etc. (2006) Study on real-time control of extraction procedure of furitus gardenia by near infrared spectroscopy. [J]. Spectroscopy and Spectral Analysis, 26(6), 1026-1030.

[6] N. L. Yang, H. B. Qu, Y. Y. Cheng, (2002) Rapid quantification of total saponins of Panax notoginseng by near infrared spectroscopy. [J]. Journal of Zhejiang University (Engineering Science), 36(4), 463-466.

[7] N. L. Yang, H. B. Qu, Y. Y. Cheng, (2003) An approach to purifying process analysis of chinese herbal extracts using NIRS. [J]. Acta Chimica Sinica., 61(5), 742-747.

[8] Z. B. Yang, L. J. Luan, (2005) Rapid determination of purifying process of Fructus Aurantii extracts by NIR transmission spectroscopy. [J]. Chinese Pharmaceutical Journal, 40(8), 615-618.

[9] Z. L. Jiang, P. Zhu, X. Qin, (2007) The new advance on pharmaceutical and clinical application of near infrared spectroscopy. [J]. Strait Pharmaceutical Journal, 19(8), 118-120.

[10] W. Z. Lu, H. F. Yuan, G. T. Xu, etc., (2000) Modern NIR technology. [M]. Bei Jing: China Petrochemical Press, 6-26.

[11] X. L. Chu, H. F. Yuan, W. Z. Lu, (2006) Research and applications of near infrared spectroscopy in China in recent years. [J]. Analytical Instrumentation, (2), 1-9. 